\title{
Considering optimal strategy in rotational atherectomy
}

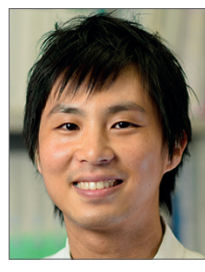

Ko Yamamoto, MD; Takeshi Kimura*, MD

Department of Cardiovascular Medicine, Kyoto University Graduate School of Medicine, Kyoto, Japan

Clinical outcomes of percutaneous coronary intervention (PCI) have been improving due to evolutions such as development of new-generation drug-eluting stents, use of intracoronary imaging device and widespread use of optimal medical therapy ${ }^{1}$.

Nevertheless, current PCI has not achieved outcomes comparable to coronary artery bypass grafting $(\mathrm{CABG})^{2-3}$, especially in patients with complex lesions such as heavily calcified lesions. Rotational atherectomy (RA) is one of the important options for heavily calcified lesions in PCI. However, the clinical adverse event rate in PCI requiring RA has been high in real-world practice ${ }^{4}$. To improve clinical outcomes of PCI requiring RA, some important procedural points should be addressed. First, avoiding life-threatening complications such as coronary perforation should be the bottom-line requirement for the appropriate practice. Second, procedural management for preventing large periprocedural myocardial injury would be essential. Previous reports have suggested that large periprocedural myocardial infarctions were associated with a substantial increase in subsequent mortality ${ }^{5}$. Third, adequate ablation for optimal stent expansion is important.
It has been reported that optimal stent expansion was associated with better clinical outcomes ${ }^{6-8}$.

The European expert consensus suggests that the safe range of speed in rotablation is between 135,000 and $180,000 \mathrm{rpm}$, because it has been reported that a speed lower than $135,000 \mathrm{rpm}$ might be associated with burr lodging, while a speed higher than $180,000 \mathrm{rpm}$ might increase platelet activation and thrombotic complications $^{9-11}$. However, in a previous randomised controlled trial comparing low-speed RA (LS-RA: 140,000 rpm) versus high-speed RA (HS-RA: 190,000 rpm), the incidence of slow flow was not significantly different ${ }^{12}$. An appropriate strategy for ablation speed still has not been established. In this issue of the Journal, Yoshida et al report an interesting technique with LS-RA $(115.000 \mathrm{rpm})$ following HS-RA $(200,000 \mathrm{rpm})^{13}$.

Article, see page 52

This study demonstrates that additional LS-RA following HS-RA ablated more plaque volume and gained a larger minimum lumen area compared with HS-RA only. There was no lodging of the burr, and slow flow occurred in five patients $(18.5 \%)$. 
Step-by-step ablation might be an important point for a safe and effective procedure. Cost-effectiveness might also be an important point. However, this is a small retrospective study. The efficacy and safety of this novel technique should be evaluated by larger randomised controlled trials. The establishment of an appropriate strategy for heavy calcification is essential for PCI evolution towards a complete alternative to $\mathrm{CABG}$.

\section{References}

1. Escaned J, Collet C, Ryan N, De Maria GL, Walsh S, Sabate M, Davies J, Lesiak M, Moreno R, Cruz-Gonzalez I, Hoole SP, West NE, Piek JJ, Zaman A, Fath-Ordoubadi F, Stables RH, Appleby C, van Mieghem N, van Geuns RJ, Uren N, Zueco J, Buszman P, Iniguez A, Goicolea J, Hildick-Smith D, Ochala A, Dudek D, Hanratty C, Cavalcante R, Kappetein AP, Taggart DP, van Es GA, Morel MA, de Vries T, Onuma Y, Farooq V, Serruys PW, Banning AP. Clinical outcomes of state-of-the-art percutaneous coronary revascularization in patients with de novo three vessel disease: 1-year results of the SYNTAX II study. Eur Heart J. 2017;38: 3124-34.

2. Farkouh ME, Domanski M, Sleeper LA, Siami FS, Dangas G, Mack M, Yang M, Cohen DJ, Rosenberg Y, Solomon SD, Desai AS, Gersh BJ, Magnuson EA, Lansky A, Boineau R, Weinberger J, Ramanathan K, Sousa JE, Rankin J, Bhargava B, Buse J, Hueb W, Smith CR, Muratov V, Bansilal S, King S, Bertrand M, Fuster V. Strategies for multivessel revascularization in patients with diabetes. N Engl J Med. 2012;367:2375-84.

3. Park SJ, Ahn JM, Kim YH, Park DW, Yun SC, Lee JY, Kang SJ, Lee SW, Lee CW, Park SW, Choo SJ, Chung CH, Lee JW, Cohen DJ, Yeung AC, Hur SH, Seung KB, Ahn TH, Kwon HM, Lim DS, Rha SW, Jeong MH, Lee BK, Tresukosol D, Fu GS, Ong TK; BEST Trial Investigators. Trial of everolimus-eluting stents or bypass surgery for coronary disease. $N$ Engl J Med. 2015;372:1204-12.

4. Fuku Y, Kadota K, Toyofuku M, Morimoto T, Ohya M, Higami H, Yamaji K, Muranishi H, Yamaji Y, Nishida K, Furukawa D, Tada T, Ko E, Ando K, Sakamoto H, Tamura T, Kawai K, Kimura T; AOI LMCA Stenting Registry Investigators. Long-Term Outcomes of Drug-Eluting Stent Implantation After Rotational Atherectomy for Left Main Coronary Artery Bifurcation Lesions. Am J Cardiol. 2019;123:1796-805.

5. Gregson J, Stone GW, Ben-Yehuda O, Redfors B, Kandzari DE, Morice MC, Leon MB, Kosmidou I, Lembo NJ, Brown WM, Karmpaliotis D, Banning AP, Pomar J, Sabate M, Simonton CA, Dressler O, Kappetein AP, Sabik JF, Serruys PW, Pocock SJ. Implications of Alternative Definitions of Peri-Procedural Myocardial
Infarction After Coronary Revascularization. J Am Coll Cardiol. 2020;76:1609-21.

6. Hong SJ, Kim BK, Shin DH, Nam CM, Kim JS, Ko YG, Choi D, Kang TS, Kang WC, Her AY, Kim Y, Hur SH, Hong BK, Kwon H, Jang Y, Hong MK; IVUS-XPL Investigators. Effect of Intravascular Ultrasound-Guided vs Angiography-Guided Everolimus-Eluting Stent Implantation The IVUS-XPL Randomized Clinical Trial. JAMA. 2015;314:2155-63.

7. Zhang JJ, Gao XF, Kan J, Ge Z, Han L, Lu S, Tian NL, Lin S, Lu QH, Wu XM, Li QH, Liu ZZ, Chen Y, Qian XS, Wang J, Chai DY, Chen CH, Li XL, Gogas BD, Pan T, Shan SJ, Ye F, Chen SL. Intravascular Ultrasound Versus Angiography-Guided Drug-Eluting Stent Implantation The ULTIMATE Trial. J Am Coll Cardiol. 2018;72:3126-37.

8. Kang SJ, Ahn JM, Song H, Kim WJ, Lee JY, Park DW, Yun SC, Lee SW, Kim YH, Lee CW, Mintz GS, Park SW, Park SJ. Comprehensive intravascular ultrasound assessment of stent area and its impact on restenosis and adverse cardiac events in 403 patients with unprotected left main disease. Circ Cardiovasc Interv. 2011;4: 562-9. 9. Barbato E, Carrie D, Dardas P, Fajadet J, Gaul G, Haude M, Khashaba A, Koch K, Meyer-Gessner M, Palazuelos J, Reczuch K, Ribichini FL, Sharma S, Sipotz J, Sjogren I, Suetsch G, Szabo G, Valdes-Chavarri M, Vaquerizo B, Wijns W, Windecker S, de Belder A, Valgimigli M, Byrne RA, Colombo A, Di Mario C, Latib A, Hamm C; European Association of Percutaneous Cardiovascular Interventions. European expert consensus on rotational atherectomy. EuroIntervention. 2015;11:30-36.

10. Reisman M, Shuman BJ, Dillard D, Fei R, Misser KH, Gordon LS, Harms V. Analysis of low-speed rotational atherectomy for the reduction of platelet aggregation. Cathet Cardiovasc Diagn. 1998;45:208-14.

11. Williams MS, Coller BS, Vaananen HJ, Scudder LE, Sharma SK, Marmur JD. Activation of platelets in platelet-rich plasma by rotablation is speed-dependent and can be inhibited by abciximab (c7E3 Fab; ReoPro). Circulation. 1998;98:742-748.

12. Sakakura K, Funayama H, Taniguchi Y, Tsurumaki Y, Yamamoto K, Matsumoto M, Wada H, Momomura S, Fujita H. The incidence of slow flow after rotational atherectomy of calcified coronary arteries: a randomized study of low speed versus high speed. Catheter Cardiovasc Interv. 2017;89:832-40.

13. Yoshida R, Ishii H, Morishima I, Tanaka A, Tsuda T, Takagi K, Morita Y, Kataoka T, Niwa K, Furusawa K, Yoshioka N, Tsuboi H, Murohara T. Ablation effect of additional low-speed rotational atherectomy following high-speed rotational atherectomy. AsiaIntervention.2020;6:52-5. 